\title{
Torsional Pile Subjected to Transient Loading in Viscoelastic Poroelastic Medium
}

\author{
Yuanqiang Cai, Gang Chen, and Feiyu Liu
}

College of Civil Engineering and Architecture, Zhejiang university, Hangzhou 310027, China

\begin{abstract}
Considering viscoelastic saturated soil, The transient dynamic response of an elastic pile is studied. The pile-soil system is divided into thin layers, the control equations of the soil are solved respectively by using Laplace transform. Considering the mixed boundary-value conditions at the interface of pile and soil, expression is derived to describe the relationship between the inner force and the displacement of the pile segment. Then the expressions of all pile segments are integrated to form the stiffness matrix of pile. Considering the transient torsional loading at the pile head, the pile displacement in time domain is calculated by numerical method, with the result showing the influence of various pile parameters.
\end{abstract}

Keywords: poroelastic medium; viscidity; elastic pile; transient dynamic response.

Wide attention has been paid to the research on the dynamic response of pile foundations. Novak [1, 2] has studied pile's torsional vibration and revealed the fundamental characteristics of the interaction between the pile and the surrounding soil. Zeng [3], Jin [4] and Cai[5] have studied the time harmonic response of elastic pile embedded in a poroelastic half-space with axial, lateral and torsional loading respectively. The analysis of pile foundation subjected to transient dynamic loading is a complex task. Militano [6] have studied the dynamic response of an elastic pile subjected to transient torsional and axial loading. It should be noted that no work on transient torsional response of a pile embedded in poroelastic soil has been reported in literature. The main

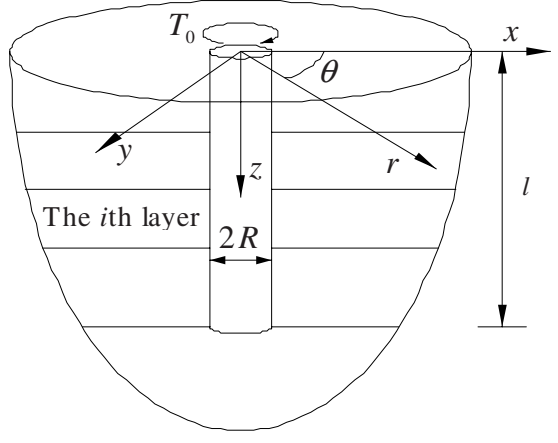

Fig. 1. Elastic pile embedded in a saturated soil objective of this paper is to present the dynamic response of an elastic pile embedded in saturated soil under transient torsional loading (Fig. 1), and the viscidity of soil is considered too. The influence of parameters of pile and soil is clarified from the numerical computation results. 


\section{Basic Equations and Solution}

The soil is divided into $k$ thin layers, and the equilibrium equation of the soil layer corresponding to torsional loading can be expressed as:

$$
\frac{\partial \sigma_{r \theta}}{\partial r}+\frac{\partial \sigma_{\theta z}}{\partial z}+2 \frac{\sigma_{r \theta}}{r}=\rho \frac{\partial^{2} u_{\theta}}{\partial t^{2}}+\rho_{w} \frac{\partial^{2} w_{\theta}}{\partial t^{2}}
$$

where $\sigma_{r \theta}, \sigma_{\theta z}$ are shear stresses of soil; $\rho=(1-n) \rho_{s}+n \rho_{w}, \rho_{s}, \rho_{w}$ signify the densities of the solid phase and the fluid phase respectively; $u_{\theta}, w_{\theta}$ denote the displacements of the solid phase and the fluid phase in the $\theta$-direction respectively.

The Voigt-Kelvin model is adopted to describe the viscidity of soil. The constitutive equations are:

$$
\begin{gathered}
\sigma_{r \theta}=G \varepsilon_{r \theta}+G^{\prime} \dot{\mathcal{E}}_{r \theta} \\
\sigma_{\theta z}=G \varepsilon_{\theta z}+G^{\prime} \dot{\mathcal{E}}_{\theta z}
\end{gathered}
$$

where $G^{\prime}$ denote the viscous parameter of the medium. $\varepsilon_{r \theta}=\frac{1}{r} \frac{\partial u_{r}}{\partial \theta}+\frac{\partial u_{\theta}}{\partial r}-\frac{u_{\theta}}{r}$, $\varepsilon_{\theta z}=\frac{\partial u_{z}}{\partial r}+\frac{\partial u_{r}}{\partial z}, \dot{\varepsilon}=\frac{\partial \varepsilon}{\partial t}$.

The equilibrium equation of the fluid can be expressed as follows:

$$
\frac{\rho_{w} g}{k_{d}} \frac{\partial w_{\theta}}{\partial t}+\rho_{w} \frac{\partial^{2} u_{\theta}}{\partial t^{2}}+\frac{\rho_{w}}{n} \frac{\partial^{2} w_{\theta}}{\partial t^{2}}=-\frac{1}{r} \frac{\partial p_{w}}{\partial \theta}=0
$$

where $k_{d}$ is the dynamic permeability coefficient containing the viscidity of liquid; $g$ denotes the gravitational acceleration; $p_{w}$ is the excess pore fluid pressure.

Militano [6] studied the transient torsional response of a pile embedded in elastic soil by neglecting the gradient of $\sigma_{r \theta}$ in the vertical direction. Following a similar assumption and considering the viscidity of soil, Equation (1) can be expressed in terms of $u_{\theta}(r, t)$ as:

$$
G\left(\frac{\partial^{2} u_{\theta}}{\partial r^{2}}+\frac{1}{r} \frac{\partial u_{\theta}}{\partial r}-\frac{u_{\theta}}{r^{2}}\right)+G^{\prime} \frac{\partial}{\partial t}\left(\frac{\partial^{2} u_{\theta}}{\partial r^{2}}+\frac{1}{r} \frac{\partial u_{\theta}}{\partial r}-\frac{u_{\theta}}{r^{2}}\right)=\rho \frac{\partial^{2} u_{\theta}}{\partial t^{2}}+\rho_{w} \frac{\partial^{2} w_{\theta}}{\partial t^{2}}
$$

The Laplace transform is introduced and its definition is given by:

$$
\widehat{f}(r, p)=\int_{0}^{\infty} f(r, t) e^{-p t} d t
$$

where $p$ is the Laplace transform parameter.

Considering Equation (4), after introducing the dimensionless constants and variables, the government equation can be expressed in the Laplace transform space:

$$
\frac{\partial^{2} \hat{\bar{u}}_{\theta}}{\partial \bar{r}^{2}}+\frac{1}{\bar{r}} \frac{\partial \hat{\bar{u}}_{\theta}}{\partial \bar{r}}-\left(s^{2}+\frac{1}{\bar{r}^{2}}\right) \hat{\bar{u}}_{\theta}=0
$$


where $s^{2}=\frac{1}{1+p \eta}\left(1+\frac{n \bar{k}_{d}}{n+p \bar{k}_{d}} \bar{\rho}_{w} p\right) p^{2}, \eta=\frac{G^{\prime}}{G}, \bar{u}_{\theta}=\frac{u_{\theta}}{R}, \bar{w}_{\theta}=\frac{w_{\theta}}{R}, \bar{r}=\frac{r}{R}$, $\bar{\rho}_{w}=\frac{\rho_{w}}{\rho}, \bar{t}=\frac{t}{R} \sqrt{\frac{G}{\rho}}, \bar{k}_{d}=\frac{k_{d}}{R g} \sqrt{\frac{G}{\rho}}$.

The general solutions of Equation (7) can be expressed as:

$$
\hat{\bar{u}}_{\theta}(\bar{r}, p)=A I_{1}(s \bar{r})+B K_{1}(s \bar{r})
$$

where $I_{n}, K_{n}$ are modified Bessel functions of the first and second kind respectively. $A(p), B(p)$ are arbitrary functions.

The soil is assumed to be infinite in the radial direction, with the displacement and stress of soil tending to be zero as $r \rightarrow \infty$. Considering the characters of the first and second kind Bessel functions, $A(p) \equiv 0$ can be identified. The displacement and stress can be expressed as:

$$
\begin{gathered}
\hat{\bar{u}}_{\theta}(\bar{r}, p)=B K_{1}(s \bar{r}) \\
\hat{\bar{\tau}}_{r \theta}(\bar{r}, p)=\frac{\partial \hat{\bar{u}}_{\theta}}{\partial \bar{r}}=-(1+p \eta) B s K_{2}(s \bar{r})
\end{gathered}
$$

The relationship between $\hat{\bar{u}}_{\theta}$ and $\hat{\bar{\tau}}_{r \theta}$ can be deduced:

$$
\hat{\bar{u}}(\bar{r}, p)=-\frac{K_{1}(s \bar{r})}{s(1+p \eta) K_{2}(s \bar{r})} \hat{\bar{\tau}}_{r \theta}(\bar{r}, p)
$$

\section{Impedance of Pile Segments and the Whole Pile}

Fig.2 shows a typical pile segment subjected to torsional loading. The dynamic response of the $i$ th pile segment is described as follows:

$$
G_{p} \frac{\partial^{2} \varphi}{\partial z^{2}}+4 \frac{\tau_{\theta}}{R^{2}}=\rho_{p} \frac{\partial^{2} \varphi}{\partial t^{2}}
$$

where $G_{p}$ and $\rho_{p}$ denote the shear modulus and mass density of pile, $\tau_{\theta}$ is the contact stress between soil and pile.

After introducing the dimensionless groups $\bar{z}=\frac{z}{R}$, $\bar{G}=\frac{G_{p}}{G}, \bar{\tau}_{\theta}=\frac{\tau_{\theta}}{G}, \bar{\rho}_{p}=\frac{\rho_{p}}{\rho}$, Equation (12) can be expressed in the Laplace transform domain as:

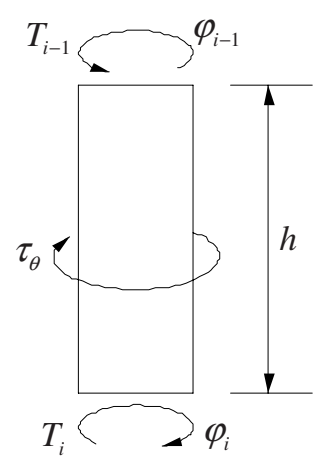

Fig. 2. Pile segment discreted 


$$
\bar{G}_{p} \frac{\partial^{2} \hat{\varphi}}{\partial \bar{z}^{2}}+4 \hat{\bar{\tau}}_{\theta}=\bar{\rho}_{p}\left(p^{2} \hat{\varphi}-p a_{i 0}-\varphi_{i 0}\right)
$$

where $a_{i 0}$ and $\varphi_{i 0}$ denote the initial values of rotational and angular velocities of the $i$ th pile segment, and are assumed to be zero. $\hat{\bar{\tau}}_{\theta}$ can be substituted by $\left.\hat{\bar{\tau}}_{r \theta}\right|_{\bar{r}_{=1}}$ of soil, and is equal to $-\left.\frac{s K_{2}(\overline{s r})}{K_{1}(s \bar{r})} \hat{\bar{u}}{ }_{\theta}(\bar{r}, p)\right|_{r=R}$. Therefore, the general solution of Equation (12) can be given as:

$$
\hat{\varphi}(\bar{z}, p)=C \exp (-\omega \bar{z})+D \exp (\omega \bar{z})
$$

where $\omega=\left\{\frac{4 s K_{2}(s)}{\bar{G}_{p} K_{1}(s)}+\frac{\bar{\rho}_{p} p^{2}}{\bar{G}_{p}}\right\}^{1 / 2} ; C(p)$ and $D(p)$ are arbitrary functions to be determined from the boundary conditions.

The relationship between the inner force and displacement of elastic pile can be expressed as $T(z)=\frac{G_{p} \pi R^{4}}{2} \frac{\partial \varphi}{\partial z}$, with the application of the Laplace transform resulting in:

$$
\hat{\bar{T}}(z, p)=\frac{\bar{G}_{p} \pi}{2} \frac{\partial \hat{\varphi}}{\partial \bar{z}}=\frac{\bar{G}_{p} \pi \omega}{2}[-C \exp (-\omega \bar{z})+D \exp (\omega \bar{z})]
$$

In the Laplace transform space, the inner forces and displacements at the segment top and bottom of the $i$ th segment are denoted as $\hat{\bar{T}}_{i-1}, \hat{\bar{T}}_{i}, \hat{\varphi}_{i-1}, \hat{\varphi}_{i}$. The substitution of the boundary condition in Equation (14) yields:

$$
\left\{\begin{array}{l}
C \\
D
\end{array}\right\}=\frac{1}{\exp (\omega \bar{h})-\exp (-\omega \bar{h})}\left[\begin{array}{cc}
\exp (\omega \bar{h}) & -1 \\
-\exp (-\omega \bar{h}) & 1
\end{array}\right]\left\{\begin{array}{c}
\hat{\varphi}_{i-1} \\
\hat{\varphi}_{i}
\end{array}\right\}
$$

Integrating Equations (15) and (16) yields the following relationship for the pile segment:

$$
\left\{\begin{array}{c}
\hat{\bar{T}}_{i-1} \\
\hat{\bar{T}}_{i}
\end{array}\right\}=\alpha\left[\begin{array}{cc}
-\beta & 2 \\
-2 & \beta
\end{array}\right]\left\{\begin{array}{c}
\hat{\varphi}_{i-1} \\
\hat{\varphi}_{i}
\end{array}\right\}
$$

where $\alpha=\frac{\bar{G}_{p} \pi \omega}{2[\exp (\omega \bar{h})-\exp (-\omega \bar{h})]}, \beta=\exp (\omega \bar{h})+\exp (-\omega \bar{h})$.

The whole pile has been discreted into $k$ segments, with Equation (17) describing the impedance of the $i$ th segment. Combining the impendance equations of all segments, the stiffness matrix in the Laplace transform domain is deduced as: 


$$
\widehat{\bar{H}}=K \widehat{\Phi}
$$

where $\widehat{\vec{H}}=\left\{\hat{\bar{T}}_{0}, 0,0, \cdots, 0, \hat{\bar{T}}_{k}\right\}^{T}$,

$$
\begin{aligned}
& \widehat{\Phi}=\left\{\widehat{\Phi}_{0}, \widehat{\Phi}_{1}, \widehat{\Phi}_{2}, \cdots, \widehat{\Phi}_{k}\right\}^{T}, \\
& K=\alpha\left[\begin{array}{ccccc}
-\beta & 2 & & & \\
-2 & 2 \beta & -2 & & \\
& \ddots & \ddots & \ddots & \\
& & -2 & 2 \beta & -2 \\
& & & -2 & \beta
\end{array}\right]_{(k+1) \times(k+1)}
\end{aligned}
$$

In Equation (18), $\hat{\bar{T}}_{0}$ is the dimensionless loading in Laplace transform domain. $\hat{\bar{T}}_{k}$ is the moment extended by soil at the bottom of the pile, and is assumed to be equal to the static stiffness corresponding to a rigid disk of radius $R$ attached to the surface of an elastic half space. Considering the viscidity of soil, the base stiffness of the pile can be expressed as $\hat{\bar{T}}_{k}=\frac{16}{3}(1+p \eta) \hat{\varphi}_{k}$ in Laplace transform domain.

Equation (18) can be solved numerically.

\section{Numerical Results and Discussions}

The solving process is carried in the Laplace transform domain. And the numerical inverse transform should be used to achieve the final result in the time domain. The numerical Laplace inversion transform method proposed by Durbin[7] is adopted in this paper:

$$
f(t)=\frac{4 e^{a t}}{S}\left[-\frac{1}{2} \operatorname{Re}\{\hat{f}(a)\}+\sum_{k=1}^{N S U M} \operatorname{Re}\left\{\hat{f}\left(a+i k \frac{2 \pi}{S}\right)\right\} \cos k \frac{2 \pi}{S} t\right]
$$

where $S, a, N S U M$ are transform parameters. The values: $S=20, a=0.75$, $N S U M=50$ are accurate enough.

Two types of loading are adopted in the numerical procedure as shown as Fig. 3, one is the rectangular pulse and the other is the triangular pulse. The non-dimensional twist angles $\varphi^{\prime}(z)=\varphi(z) G / T_{0}$ are calculated to illustrate the influence of various factors. Some properties of poroelastic material and pile are $n=0.4, \rho_{s}=2650 \mathrm{~kg} / \mathrm{m}^{3}$, $\rho_{w}=1000 \mathrm{~kg} / \mathrm{m}^{3}, \bar{\rho}_{b}=1.3, \bar{t}_{0}=2$.

Fig. $4\left(l / R=15, k_{d}=10^{-7} \mathrm{~m} / \mathrm{s}, \eta=0\right)$ shows the displacement of the pile head in the time domain, part (a) and part (b) show the dynamic responses of elastic pile with different $\bar{G}_{p}$ under the rectangular pulse and the triangular pulse respectively ( $\varphi_{0}^{\prime}$ denotes the non-dimensional displacement of the pile head). The results indicate 


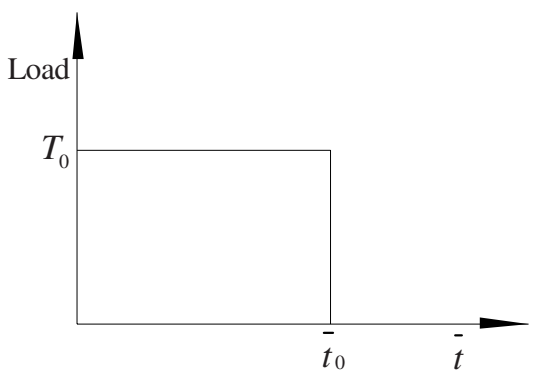

(a) rectangular pulse

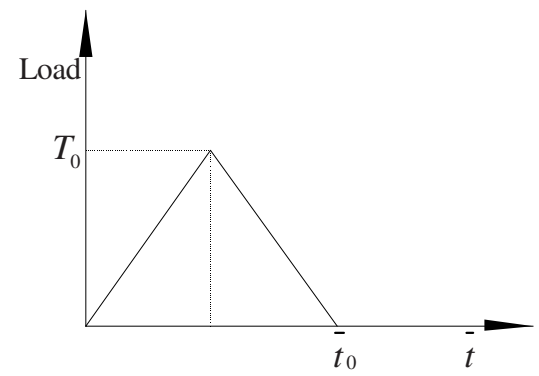

(b) triangular pulse

Fig. 3. Transient load typses

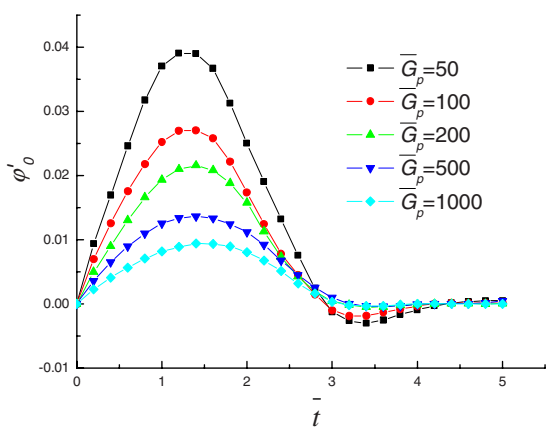

(a) Response under rectangular pulse

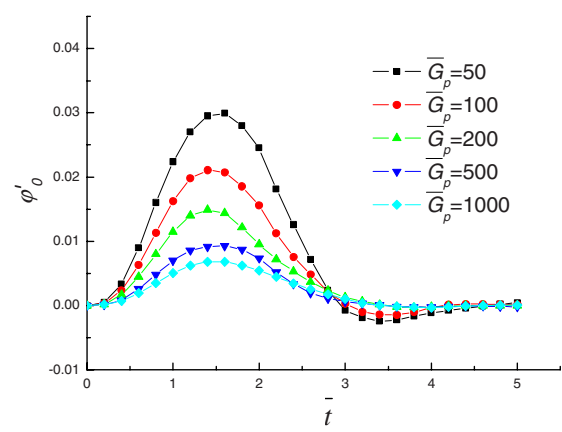

(b) Response under triangular pulse

Fig. 4. Time histories of the non-dimensional twist angle of the pile head with different shear modulus

that the displacement is oscillating in the time domain, and is close to zero finally. Each curve has an obvious peak value varying with different $\bar{G}_{p}$. The pile head displacement increases with the reduction of the pile's Young's modulus. Under two types of loading, the response curves are alike. But the pile head displacement is small under triangular pulse, and the trends are different at the initial stages.

The displacement responses of the surrounding soil for different shear modulus of pile are also taken into consideration. Time histories of the non-dimensional displacement of soil around the pile are depicted in Fig.5. $\left(k_{d}=10^{-7} \mathrm{~m} / \mathrm{s}, \eta=0\right)$. It's found that a flexible pile $\left(\bar{G}_{p}=100\right)$ causes larger soil responses than a stiffer pile $\left(\bar{G}_{p}=500\right)$. And for a stiffer pile, the generated surface wave can not propagate as far as that of a flexible pile.

By changing the soil viscidity, computation results of the non-dimensional twist angle at the pile head are presented in Fig. 6 and Fig. $7\left(k_{d}=10^{-7} \mathrm{~m} / \mathrm{s}\right)$ for $\bar{G}_{p}$ with 


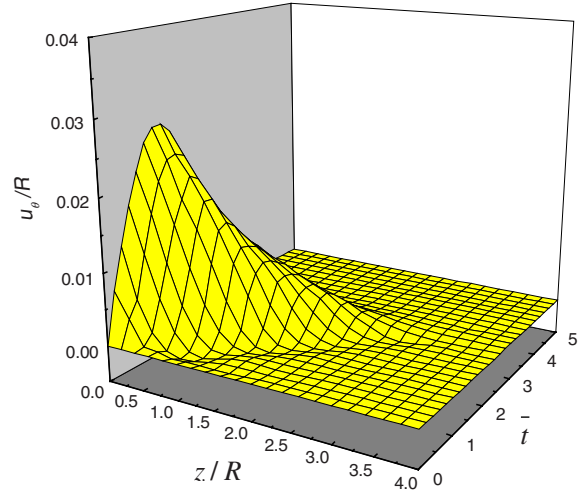

(a) Response with $\bar{G}_{p}=100$

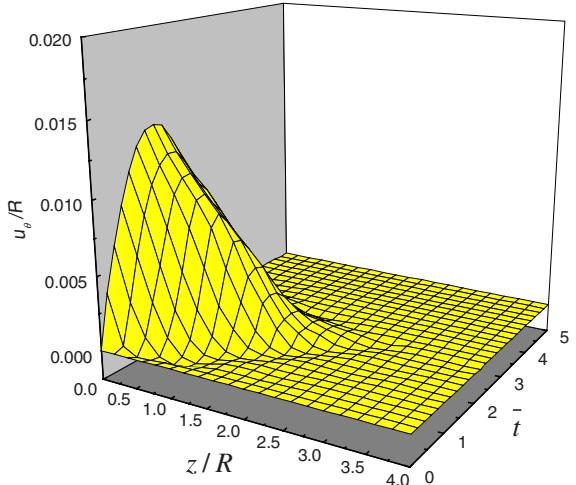

(b) Response with $\bar{G}_{p}=500$

Fig. 5. Time histories of the non-dimensional displacement of soil around the pile

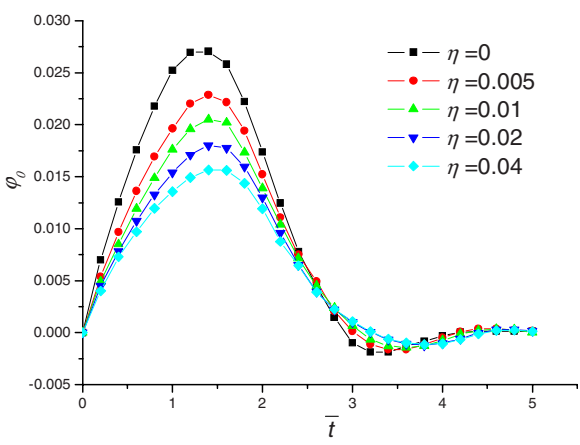

(a) $\quad l / R=10$

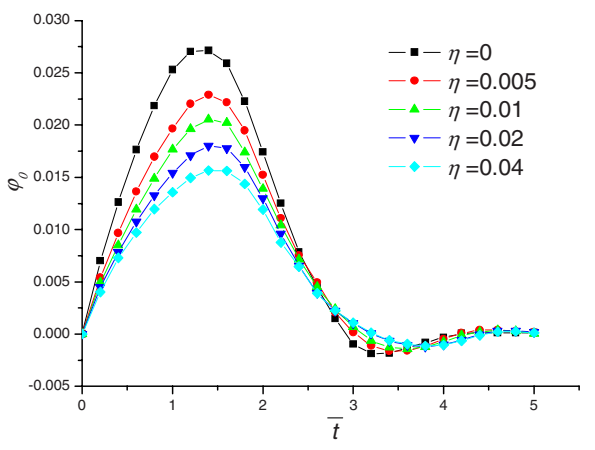

(b) $l / R=30$

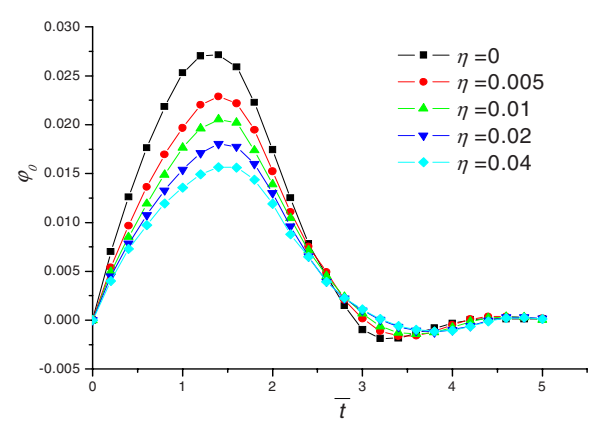

(c) $\quad l / R=50$

Fig. 6. Time histories of the non-dimensional twist angle of the pile head with different $\operatorname{viscidity}\left(\bar{G}_{p}=100\right)$ 


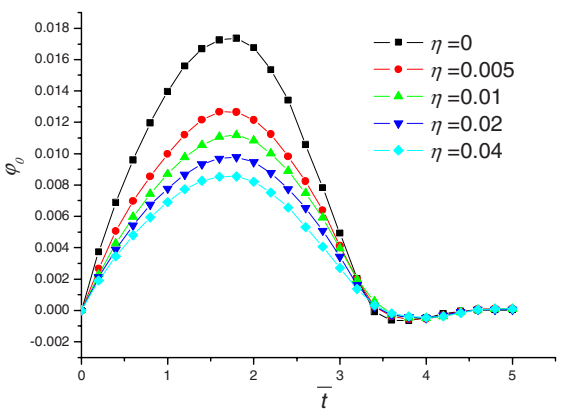

(a) $l / R=10$

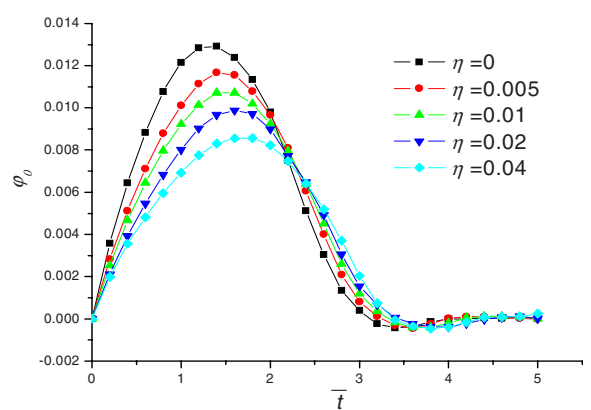

(b) $\quad l / R=30$

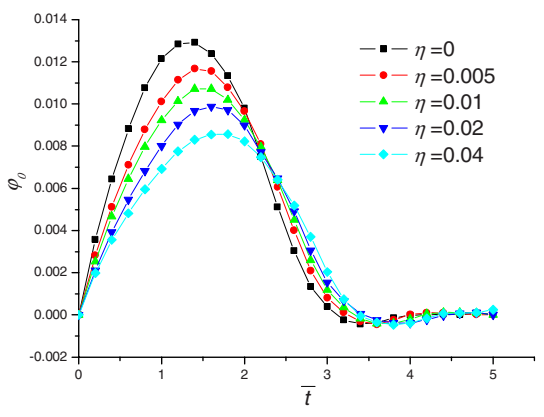

(c) $\quad l / R=50$

Fig. 7. Time histories of the non-dimensional twist angle of the pile head with different viscidity $\left(\bar{G}_{p}=500\right)$

values of 100 and 500 respectively. The results show that the soil viscidity remarkably affects the response amplitudes at the pile head, and the bigger value of $\eta$ leads to the smaller amplitude of twist angle. Also, it is found that the increasing of the soil viscidity causes a time lag for the occurrences of response peaks at pile head. In the range of $\eta$ taken into account, only when the pile is stiff ( $\bar{G}_{p}=500$ ) the change of slenderness ratio of pile will cause a difference in the response that can be noticed.

\section{Conclusion}

Analytical method was developed to study the transient dynamic response of an elastic pile embedded in a homogeneous poroelastic medium and subjected to transient torsional loading. Numerical method was used to calculate the elastic pile twist angle, and the conclusions were obtained as follows:

1) Compared with the triangular load, the rectangular step torque causes larger pile displacement.

2) The non-dimensional shear modulus affects substantially the pile transient response, the peak twist angle of pile decreases with the increasing of pile stiffness. 
3) The flexible pile contributes more to the surrounding surface soil than the stiffer pile.

4) The bigger value of $\eta$ leads to the smaller amplitude of twist angle, and the increasing of soil viscidity causes a time lag for the occurrences of response peaks at the pile head.

\section{Acknowledgements}

The work presented in this paper was supported by the National Natural Science Foundation of China, under Grant number 50478081.

\section{References}

1. M. Novak and J.F. Howell, Torsional Vibration of Pile Foundations. Journal of the Engineering Mechanics Division, 103. (1977) 271-285

2. M. Novak and J.F. Howell, Dynamic Response of Pile Foundations. Journal of the Engineering Mechanics Division, 104. (1978) 535-552

3. Zeng and R.K.N.D. Rajapakse, Dynamic Axial Load Transfer from Elastic Bar to Proelastic Medium. Journal of the Engineering Mechanics Division, 125. (1999) 1048-1055

4. B. Jin, D. Zhou and Z. Zhong, Lateral Dynamic Compliance of Pile Embedded in Poroelastic Half-Space. Soil Dynamics and Earthquake Engineering, 21. (2001) 519-525

5. Y.Q. Cai, G. Chen and C.J. Xu et. al, Torsional response of pile embedded in a poroelastic medium. Soil Dynamic and Earthquake Engineering, 26. (2006) 1143-1148

6. G. Militano and R.K.N.D. Rajapakse, Dynamic Response of a Pile in a Multi-layered Soil to Transient Torsional and Axial Loading. Geotechnique, 49. (1999) 91-109

7. F. Durbin, Numerical inversion of Laplace transforms: an efficient improvement to Dubner and Abate's method. The Computer Journal, 17. (1974) 371-376 